C-A/AP/\#140

March 2004

\title{
Path Length through Helical Snakes and Rotators
}

\author{
W. MacKay
}

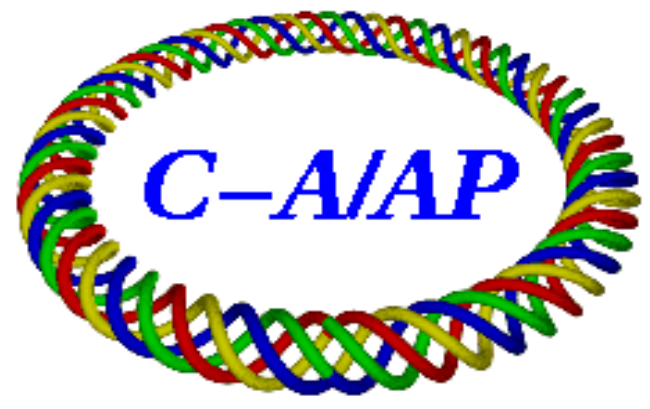

Collider-Accelerator Department Brookhaven National Laboratory Upton, NY 11973 
Path Length through Helical Snakes and Rotators

Waldo MacKay

4 Mar., 2004

The magnetic field in a helical magnet of a RHIC snake has the approximate form

$$
\begin{aligned}
& B_{x}=-B_{0} \sin (k z), \\
& B_{y}=B_{0} \cos (k z), \\
& B_{z}=0
\end{aligned}
$$

to lowest order, where $k=2 \pi / \lambda$ with $\lambda$ being the wavelength of the helical twist of the magnet's coil. The twist of the magnet produces higher order terms off-axis, but we should be able to ignore this for a reasonable estimate of path length. From the Lorentz force we obtain the approximate equations of motion[1]

$$
\begin{aligned}
x^{\prime \prime} \approx-\frac{q}{p} B_{y} & =-\frac{q B_{0}}{p} \sin (k z), \\
y^{\prime \prime} \approx \frac{q}{p} B_{x} & =\frac{q B_{0}}{p} \cos (k z) .
\end{aligned}
$$

Integrating once gives the equations:

$$
\begin{aligned}
& x^{\prime}(s) \approx \frac{q B_{0}}{p k} \cos (k z)+C_{1}, \\
& y^{\prime}(s) \approx \frac{q B_{0}}{p k} \sin (k z)+C_{2} .
\end{aligned}
$$

For the design orbit solution we have initial angles of $x^{\prime}(0)=y^{\prime}(0)=0$, so $C_{1}=-q B_{0} /(p k)$ and $C_{2}=0$ :

$$
\begin{aligned}
& x^{\prime}(s) \approx \frac{q B_{0}}{p k}[\cos (k z)-1], \\
& y^{\prime}(s) \approx \frac{q B_{0}}{p k} \sin (k z) .
\end{aligned}
$$

So the path length through the helix should be

$$
\begin{aligned}
s & =\int_{0}^{\lambda} \sqrt{1+\left(x^{\prime}\right)^{2}+\left(y^{\prime}\right)^{2}} d z \\
& \approx \int_{0}^{\lambda} \sqrt{1+2\left(\frac{q B_{0}}{p k}\right)^{2}[1-\cos (k z)]} d z
\end{aligned}
$$


which leads to an elliptic integral of the second kind. However, we may expand the square root in a Taylor series to approximate the integral:

$$
\begin{aligned}
s & \approx \int_{0}^{\lambda}\left[1+\left(\frac{q B_{0}}{p k}\right)^{2}[1-\cos (k z)]\right] d z \\
& =\lambda\left[1+\left(\frac{q B_{0}}{p k}\right)^{2}\right] .
\end{aligned}
$$

The increase in path length through the helix is then

$$
\begin{aligned}
\Delta s & =s-\lambda \\
& \approx \frac{\lambda^{3}}{4 \pi^{2}}\left(\frac{q B_{0}}{p}\right)^{2} .
\end{aligned}
$$

For the full snake (or rotator) the increase is

$$
\Delta s \approx \frac{\lambda^{3}}{2 \pi^{2}}\left(\frac{q}{p}\right)^{2}\left[B_{\mathrm{in}}^{2}+B_{\mathrm{out}}^{2}\right]
$$

where $B_{\text {in }}$ and $B_{\text {out }}$ are the fields in the inner and outer pairs of helices of the snake.

At injection $(G \gamma=46.5), p / q=81.1138 \mathrm{Tm}$. Each helix is about $2.41 \mathrm{~m}$ long, and with the snake fields at $B_{\text {out }}=1.26 \mathrm{~T}$ and $B_{\text {in }}=4.03 \mathrm{~T}$ this gives an increase in path length of $1.92 \mathrm{~mm}$ per snake. At $100 \mathrm{GeV} \Delta s$ decreases to $0.11 \mathrm{~mm}$. Vahid's calculation gave an increase of $1.9338 \mathrm{~mm}$ at $G \gamma=46.5$ (injection) which agrees with my estimate. To write a simple scaling law for general settings of the RHIC snakes and rotators, we can write

$$
\begin{aligned}
\Delta s & =\left[\left(\frac{46.5}{1.7928474}\right)^{2}-1\right] \frac{1.9338 \mathrm{~mm}}{\gamma^{2}-1} \\
& =0.0123\left[\mathrm{~m} / \mathrm{T}^{2}\right] \frac{B_{\text {out }}^{2}+B_{\text {in }}^{2}}{\gamma^{2}-1} \text { per snake or rotator. }
\end{aligned}
$$

A simple cubic fit to field vs current in the RHIC helices which is good to better than $0.5 \%$ is given by

$$
B(I)=0.0035+(0.013) I+\left(6.3136 \times 10^{-6}\right) I^{2}-\left(2.341 \times 10^{-8}\right) I^{3}
$$

with $B$ in tesla and $I$ in amperes. I do have a much more accurate algorithm for $B$ vs $I$, but it is considerably more complicated than needed here.

Having discussed this with Mike Brennan, it seems that the simplest way to handle the path length increase for each ring would be to use an ADO parameter to specify a single constant

$$
C=0.0123\left[\mathrm{~m} / \mathrm{T}^{2}\right] \sum_{j \in \mathcal{N}}\left[B_{\mathrm{out}, j}^{2}+B_{\mathrm{in}, j}^{2}\right]
$$


where $\wedge$ is the set of snakes and rotators in the ring. The ADO parameter may be set by the sequencer, since we will not be ramping snakes and rotators at the same time as changing the energy. The total path length can then be calculated by the DSP via the simple formula

$$
\Delta s=\frac{C}{\gamma^{2}-1} .
$$




\section{AGS helices:}

In the AGS the new helical dipoles have three sections of two different pitches none of which twist a complete revolution. The path length may be calculated by starting from Eq. (1) and writing the corresponding indefinite integral as

$$
\begin{aligned}
I(\theta) & \approx \int\left[1+\left(\frac{q B_{0}}{p k}\right)^{2}[1-\cos (k z)]\right] d z \\
& =\lambda\left[1+\left(\frac{q B_{0}}{p k}\right)^{2}\right] . \\
& =\frac{\theta}{k}+k^{-3}\left(\frac{q B_{0}}{p}\right)^{2}[\theta-\sin \theta],
\end{aligned}
$$

where $\theta=k z$ is measured in the transverse plane away from the vertical. So to estimate the path length increase we just sum over the various helical segments and subtract the total length of the snake:

$$
\Delta s \approx\left(\frac{q}{p}\right)^{2} \sum_{j=1}^{3} \frac{B_{j}^{2}}{k_{j}^{3}}\left[\theta_{j, 2}-\theta_{j, 1}-\sin \theta_{j, 2}+\sin \theta_{j, 1}\right] .
$$

A rough estimate of $\Delta s$ for the AGS warm snake is given in the following table:

Parameters for the AGS Warm Helical Snake at $\gamma=4.5$

$\begin{array}{rrrrrcc}z_{j, 1}[\mathrm{~m}] & z_{j, 2}[\mathrm{~m}] & \theta_{j, 1} & \theta_{j, 2} & k\left[\mathrm{~m}^{-1}\right] & B[\mathrm{~T}] & \Delta s_{j}[\mathrm{~mm}] \\ -0.98 & -0.64 & -249.5^{\circ} & -128.7^{\circ} & 6.20 & 1.3 & 0.52 \\ -0.64 & 0.64 & -128.7^{\circ} & 128.7^{\circ} & 3.51 & 1.5 & 2.94 \\ 0.64 & 0.98 & 128.7^{\circ} & 249.5^{\circ} & 6.20 & 1.3 & 0.52 \\ & & & & & \Delta s: & 3.98\end{array}$

Alfredo's[2] more careful integration gives a $3.46 \mathrm{~mm}$ increase in path length. The scaling $\Delta s \propto p^{-2}$ still agrees quite well with his estimates through the whole AGS energy range.

For the cold snake[2], I calculate a path length increase of $1.50 \mathrm{~cm}$ using Alfredo's numbers (his Table 2 on p. 6). His integration gives only $1.19 \mathrm{~cm}$. His values for different energies scale well with $p^{-2}$. The additional low-field solenoid in middle of the may perturb things a bit, but probably not that much. For the cold snake I would suggest using a calculation for a given current setting which integrates the trajectory through the field map and then scale it with $p^{-2}$. We should probably integrate the field maps for several current settings to get the current scaling right.

\section{References}

1. E. D. Courant, "Orbit Matrices for Helical Snakes", AGS/RHIC/SN No. 004

2. Alfredo U. Luccio, "Tracking through a Warm Helical Snake for the AGS", CA/AP/\#136 (2004). 